\title{
A Rare Case of Spontaneous Asymptomatic Renal Artery Pseudoaneurysm Treated with Coil Embolization: A Case Report
}

\author{
Dinesh Chataut $^{1}$ Santosh Maharjan ${ }^{1}$ Om Biju Panta ${ }^{2}$ Ram Kumar Ghimire ${ }^{1}$ \\ ${ }^{1}$ Department of Radiology and Imaging, Tribhuwan University \\ Teaching Hospital, Maharajgunj, Kathmandu, Nepal \\ 2 Department of Radiology and Imaging, Koshi Zonal Hospital, \\ Biratnagar, Nepal \\ Address for correspondence Om Biju Panta, MD, Department of \\ Radiology and Imaging, Koshi Zonal Hospital, Rangeli Road, Biratnagar \\ 56700, Nepal (e-mail: bijupanta@yahoo.com). \\ J Clin Interv Radiol ISVIR 2017;1:56-60.
}

\begin{abstract}
Background Renal artery pseudoaneurysm (RAP) is a rare renal vascular abnormality but a life-threatening condition that requires multiple imaging modalities for diagnosis and successful management. It can occur as a complication associated with a percutaneous renal biopsy procedure, renal surgery, and trauma. Asymptomatic spontaneous RAP is also a rare entity and not reported in literature.

Case Presentation A 28-year-old patient presented to the outpatient department for ultrasound of the breast for mastalgia and routine ultrasound of the abdomen. Ultrasound breast was normal; however, a cystic lesion with color uptake with a yinyang pattern and to-and-fro spectral waveform was seen in the lower pole of left kidney suggesting a pseudoaneurysm. A computed tomography renal angiogram confirmed the diagnosis. The patient was completely asymptomatic with no history of any renal surgery or intervention or any trauma to the abdomen. Her general and systemic

Keywords

- renal artery pseudoaneurysm

- angiogram

- embolization

- case report examinations were unremarkable. Digital subtraction angiography and coil embolization of the pseudoaneurysm were performed. Absent of flow in the lesion was demonstrated in postembolization angiography images and ultrasound images. Conclusion RAP has always been described in patients after renal biopsy, surgery, interventions, or trauma. However, we present a case of asymptomatic spontaneous RAP, which was incidentally discovered and effectively treated with coil embolization.
\end{abstract}

\section{Background}

Renal artery pseudoaneurysm (RAP) is an uncommon but potentially life-threatening condition that is often difficult to diagnose. Typically, RAPs are seen in patients after renal biopsy, percutaneous nephrolithotomy, partial nephrectomy, or trauma. Most of the time, small pseudoaneurysms are asymptomatic and thrombose spontaneously. ${ }^{1}$ However, when they are symptomatic, patient can present with a pulsatile mass in the pelvis, flank pain, renal dysfunction secondary to compression of artery branches or shunting of blood, or hemorrhage from rupture. Although these lesions can be managed surgically, renal artery embolization is preferred if they are amenable to embolization to minimize the loss of normal renal parenchyma. ${ }^{2,3}$ Spontaneous pseudoaneurysm of segmental renal artery is also a rare entity. Here we present a case of asymptomatic spontaneous segmental RAP, which was diagnosed incidentally and subsequently coil embolization was performed.

\section{Case Presentation}

A 28-year-old female was referred to the Department of Radiology for ultrasonography (USG) of breast for mastalgia and routine USG of abdomen/pelvis. Her breast USG was normal. In USG of the abdomen, there was a well-defined 


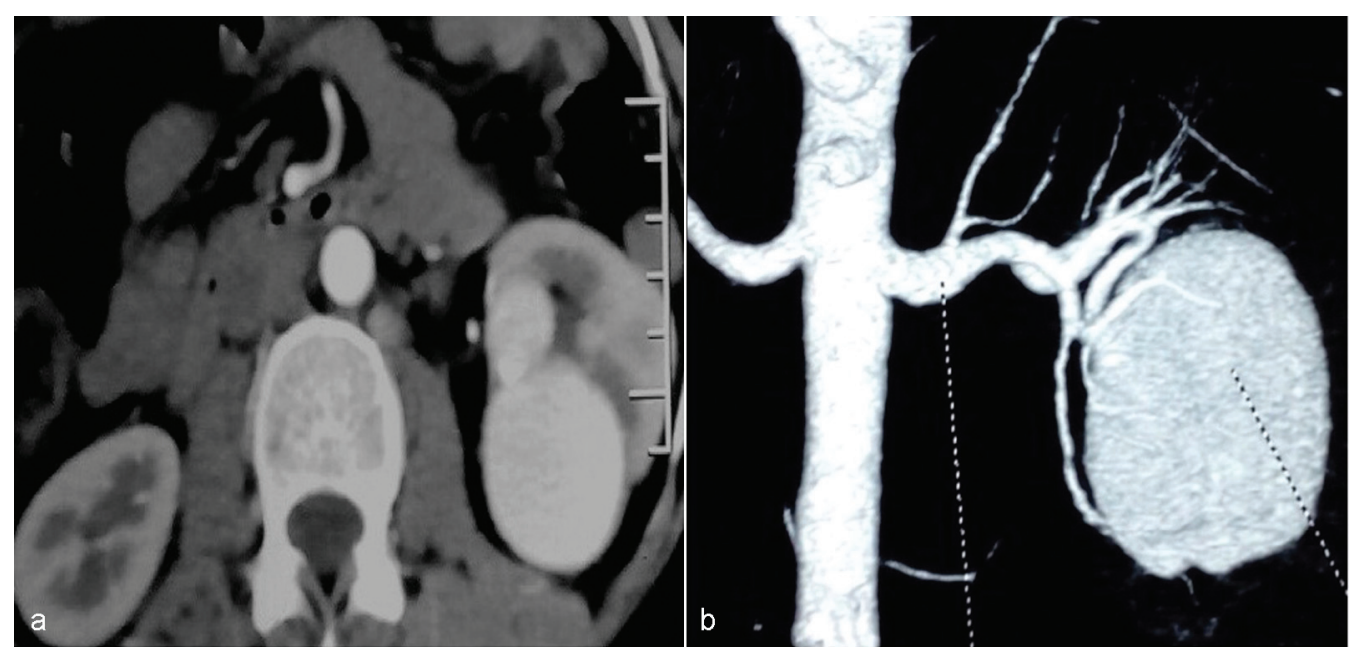

Fig. 1 (a) Axial contrast computed tomography of abdomen and three-dimensional volume rendering images. (b) A pseudoaneurysm in the lower pole of the left kidney arising from the posterior segmental renal artery (arrows).

thin-walled cystic lesion approximately $4.8 \times 3 \mathrm{~cm}$ in size in the lower pole of the left kidney. On color Doppler study, the lesion showed "to-and-fro" pattern of color flow within, giving rise to the characteristic "yin-yang pattern," highly suggestive of pseudoaneurysm. Computed tomography (CT) renal angiography was performed, which showed a pseudoaneurysm with a size of approximately $4.8 \times 3.5 \times 3.3 \mathrm{~cm}$ in the lower pole of the left kidney, arising from the posterior segmental branch of the left renal artery ( - Fig. 1 ). There was no evidence of arteriovenous fistula. All other abdominopelvic organs, as well as the aorta and rest of the arteries were normal.

Clinical examination revealed no abnormality. Her blood pressure was 110/80 $\mathrm{mm} \mathrm{Hg}$ and her pulse was 62 beats per minute. Blood urea nitrogen was $14.1 \mathrm{mg} / \mathrm{dL}$ and serum creatinine was $0.79 \mathrm{mg} / \mathrm{dL}$. Hemoglobin was $14.3 \mathrm{~g} / \mathrm{dL}$, hematocrit was $46.1 \%$, and platelets were $2,22,000 / \mu \mathrm{L}$. Prothrombin time was 11.0 seconds and activated partial thromboplastin time was 34.2 seconds. On urine analysis, proteinuria and hematuria were not present $(0-2$ red blood cells per high power field). She had caesarian section 3 years ago for fetal distress, which was uneventful. There was no history of systemic illness, hematuria, joint pain, skin rashes, recent fever, renal surgery, renal biopsy, or abdominal trauma.

She was then planned for digital subtraction angiography (DSA) and coil embolization. Selective renal angiogram revealed a large pseudoaneurysm in the lower pole of the left kidney, arising from the posterior segmental branch of the left renal artery (-Fig. 2a). The posterior segmental artery was selectively catheterized and $3 \times 3 \mathrm{~mm}$ coil was deployed at the distal part of the artery. Repeat renal angiogram after 1 minute was performed, which revealed complete thrombosis of the distal part of the posterior segmental artery with the absence of contrast opacification of the pseudoaneurysm ( $\mathbf{- F i g}$. 2b). There were no intraoperative and postoperative complications. Repeat renal Doppler USG was performed the



Fig. 2 (a) Selective posterior segmental angiogram showing a large pseudoaneurysm arising from posterior segmental artery in lower pole of left kidney. (b) Angiography after embolization using a coil shows a well-occluded feeding artery. The absence of filling of the pseudoaneurysm with contrast material indicates successful embolization. 




Fig. 3 Follow-up color Doppler ultrasonography showing complete absence of flow in the pseudoaneurysm along with increased echogenicity within indicating successful embolization and complete thrombosis.

next day, which showed complete absence of flow in the pseudoaneurysm, with increased internal echogenicity suggesting thrombosis (-Fig. 3). Her hospital stay was uneventful and she was discharged after 24 hours of hospital stay.

\section{Discussion}

RAP is a rare clinical entity that may occur after renal biopsy, renal surgery, renal transplantation, penetrating trauma, or blunt renal trauma. Passage of blood from a lacerated renal artery to the renal parenchyma leads to the formation of RAP. ${ }^{1,2}$ After renal injury, combination of hypotension, coagulation, and pressure from the surrounding tissues, such as the vascular adventitia, renal parenchyma, and Gerota's fascia, result in temporary cessation of the bleeding. Later dissolution of the clot and surrounding necrotic tissue results in recanalization between the intravascular and extravascular space, which ultimately leads to formation of a pseudoaneurysm. With restoration of normal blood flow in the artery, the pseudoaneurysm can grow in size and eventually become unstable, with erosion into the surrounding perinephric tissue.,

However, asymptomatic spontaneous RAP as in our case, without history of surgery, renal biopsy, significant abdominal trauma, and systemic disease, is a very rare entity. Previous trivial abdominal trauma may also be a predisposing factor for the development of renal RAP and the patient may not elicit history of such minor event. Another cause of spontaneous RAP can be vasculitides such as polyarteritis nodosa. Renal involvement in polyarteritis nodosa often leads to arterial hypertension and ischemic nephropathy with renal insufficiency. Imaging studies may show renal parenchymal infarcts with stenoses and microaneurysms, which can rupture and cause hematomas. With subsequent resolution of intrarenal hematoma, as in case of renal trauma, there is high chance of developing pseudoaneurysms. ${ }^{5}$

Symptoms of RAP may include abdominal tenderness, abdominal mass, hematuria, hypertension, and shock. Signs and symptoms of RAP may develop immediately after the insult or they may be delayed. ${ }^{6}$ Hematuria is the most common symptom associated with RAP and results from the erosion of RAP into the adjacent renal collecting system; this may occur within 2 to 4 weeks after the injury. ${ }^{7}$ A metaanalysis including 13 studies of partial nephrectomy, which included a total of 105 patients with RAP, showed that 102 (97\%) patients were symptomatic at presentation. The most common symptoms at presentation were gross hematuria, flank pain, and anemia, with gross hematuria in $87.3 \%$ of patients. Along with hematuria, flank pain and anemia were commonly reported, but only $5 \%$ of patients presented with all three symptoms. RAP symptoms presented at a mean of postoperative day 14.9 (range: $1-90){ }^{8}$

However, patients can present with nonspecific symptoms and therefore RAP is incidentally found. In one case, the patient presented with hematuria only, and arteriovenous fistula and pseudoaneurysm were diagnosed 10 years after kidney allograft biopsy. ${ }^{9}$ Asymptomatic RAP has also been detected 4 years after renal biopsy. ${ }^{10}$

Completely asymptomatic RAP without history of renal surgery or trauma is very rare and even more difficult to diagnose. As in our case, the diagnosis was made incidentally during routine USG of the abdomen and pelvis. Therefore, it is difficult to diagnose an RAP without a high index of suspicion. These findings suggest that after renal biopsy, renal surgery, or abdominal trauma, it is necessary to periodically evaluate the kidney using imaging studies.

In fact, the diagnosis of RAP is challenging. Angiography has been the standard tool for diagnosis. However, if the patient is stable, noninvasive tests such as contrast medium-enhanced $\mathrm{CT}$, color Doppler sonography, or magnetic resonance angiography should be performed. ${ }^{11} \mathrm{CT}$ angiography has advantage of imaging of the entire urinary tract and is the technique of choice for follow-up. ${ }^{12}$ RAP is best seen on the arterial phase, which appears as a focal high attenuation lesion with a density similar to that of the adjacent arterial vessels. RAP may also be visible in the nephrographic phase, but may be missed when the adjacent renal parenchyma is densely enhanced, masking the high density of the pseudoaneurysm. Due to washout of contrast material from the pseudoaneurysm in pyelographic phase, RAP will not be seen. ${ }^{13}$

Pseudoaneurysm resembles a cystic mass on sonography. The ultrasound findings may not be sufficient to distinguish a hematoma from a pseudoaneurysm. Color Doppler sonography is very useful and shows characteristic to-and-fro flow within the lesion. If imaging findings are not conclusive and there is clinical suspicion of RAP, or the patient is hemodynamically unstable, angiography should be undertaken. In addition to high sensitivity in identifying RAP, the advantages of angiography include the potential to achieve simultaneous endovascular management of RAP. ${ }^{14}$ Embolization of RAP has been reported to have high success rates exceeding $90 \%{ }^{4,14-17}$ The risk of rupture is estimated to be low, but is associated with a death rate as high as $80 \%{ }^{18}$

Currently, no definite guidelines exist for the size at which an RAP should be repaired in an asymptomatic patient. In general, aneurysms greater than $2 \mathrm{~cm}$ in diameter are considered to have a high risk of rupture. ${ }^{19}$ In fact, it has been reported that most pseudoaneurysms are small and asymptomatic and sometimes resolve spontaneously. ${ }^{20}$ 
However, growth of the pseudoaneurysm may occur rapidly. A previous report showed diameter evolving from 17 to $30 \mathrm{~mm}$ within 2 months. ${ }^{21}$ Another report demonstrated that a pseudoaneurysm was diagnosed at $30 \mathrm{~mm}$, whereas a CT scan performed 2 months before was normal. ${ }^{22}$ Given that ruptures have also been reported in smaller aneurysms if left untreated, it was decided that an intervention on the RAP would decrease the risk of ultimate rupture. ${ }^{23}$

In our case, the size of the pseudoaneurysm was larger and was arising from a large posterior segmental renal artery. Though asymptomatic, owing to its large size and high risk of rupture, team of intervention radiologist and urosurgeons decided to intervene in the pseudoaneurysm by percutaneous coil embolization.

Management methods of RAP are also a challenging issue, and a variety of treatment modalities have been exploited so far. ${ }^{7}$ Treatment of RAP can be by nephrectomy, open vascular surgery, or angiographic embolization, depending on the patient's clinical condition. Surgical indications for repair include overt ruptures, an aneurysm greater than $2 \mathrm{~cm}$, renovascular hypertension, expansion of the aneurysm, and evidence of renal damage. ${ }^{7}$

In general, angiographic embolization is the procedure of choice for management of RAP due to its minimally invasive and selective nature and the maximal preservation of renal parenchyma. ${ }^{6}$ Renal artery embolization begins with a renal angiogram through a transfemoral approach. Selective embolization of renal artery branches can be achieved by using microcatheters inserted coaxially over a guidewire. ${ }^{16}$ The embolization material should be chosen based on the patient's vascular anatomy and the specific clinical indication or pathologic process necessitating the procedure. Resorbable materials, coils, inert particles, and sclerosants (liquids) can be used, depending on the clinical indication and vascular structure to be occluded. ${ }^{16,17}$ Many case series and reports have shown that coil embolization is an effective method for treating renal pseudoaneurysms following different cause with high success rates. ${ }^{7,10,19,24-27}$ The general goal is to occlude the branch with hemorrhagic extravasation while sparing surrounding branches to limit parenchymal damage.

However, embolization for the management of RAP appears to have some shortcomings such as possible reflux of embolic material into the normal proximal vessel if the distal branch has not been selectively cannulated and the risk of more generalized ischemia resulting from thrombosis of a main feeding branch. ${ }^{7,16,17}$ To overcome these limitations, treatment with covered stent grafts on RAP located in branches of visceral arteries has been suggested. ${ }^{14}$ In selected cases, the use of ultrasound-guided thrombin injection into an extracapsular pseudoaneurysm following renal allograft biopsy can also be considered a treatment option. ${ }^{28}$

\section{Conclusion}

In conclusion, we herein showed a case of a 28-year-old woman with spontaneous asymptomatic segmental RAP diagnosed incidentally during routine USG of the abdomen, which was later confirmed with CT renal angiography and DSA of the renal artery. RAP was treated with percutaneous selective coil angioembolization of the posterior segmental renal artery, and follow-up imaging showed successful resolution without evidence of complications. Clinicians and radiologist need to bear in mind that even without history of major renal trauma or surgery, renal pseudoaneurysm can occur in asymptomatic individuals. Renal artery embolization is a minimally invasive technique for the diagnosis and management of RAPs. Unlike other imaging modalities, such as CT and USG, angiography not only demonstrates the anatomic location of pseudoaneurysms, but also allows the interventional radiologist to quickly and effectively treat this potentially life-threatening complication. Our cases demonstrate the efficacy of this technique.

\section{Declaration \\ Consent and ethical approval were obtained.}

Conflicts of Interest

None.

\section{Competing Interest}

The authors declare that they have no competing interests.

Funding

None.

\section{Authors Contribution}

Dinesh Chataut conceived the case report and was actually involved in case management, acquiring the CT angiogram and DSA images, and assisted in drafting the article and reviewing and finalizing the manuscript. Santosh Maharjan and Ram Kumar Ghimire were actually involved in case management, acquiring the CT angiogram and DSA images, and assisted in drafting the article and reviewing and finalizing the manuscript. Om Biju Panta conceived the study, designed the case report, and drafted the case report, revising it several times before finalizing and approving the manuscript. He is the corresponding author for the case study.

Note

No other datasets were used apart from the patient images that are present in this article.

\section{References}

1 Nakatani T, Uchida J, Han YS, et al. Renal allograft arteriovenous fistula and large pseudoaneurysm. Clin Transplant 2003;17(1):9-12

2 Sauk S, Zuckerman DA, Eds. Renal artery embolization. Seminars in interventional radiology; hieme Medical Publishers; 2011

3 Srivastava A, Singh KJ, Suri A, et al. Vascular complications after percutaneous nephrolithotomy: are there any predictive factors? Urology 2005;66(1):38-40 
4 Lee RS, Porter JR. Traumatic renal artery pseudoaneurysm: diagnosis and management techniques. J Trauma 2003;55(5): 972-978

5 Hachulla E, Bourdon F, Taieb S, et al. Embolization of two bleeding aneurysms with platinum coils in a patient with polyarteritis nodosa. J Rheumatol 1993;20(1):158-161

6 Mima A, Toma M, Matsubara T, et al. Angio-embolization of renal artery pseudoaneurysm after renal biopsy: a case report. Ren Fail 2009;31(8):753-755

7 Albani JM, Novick AC. Renal artery pseudoaneurysm after partial nephrectomy: three case reports and a literature review. Urology 2003;62(2):227-231

8 Jain S, Nyirenda T, Yates J, Munver R. Incidence of renal artery pseudoaneurysm following open and minimally invasive partial nephrectomy: a systematic review and comparative analysis. J Urol 2013;189(5):1643-1648

9 Jin KB, Hwang EA, Han SY, et al, eds. Delayed presentation of arteriovenous fistula and pseudoaneurysms in a renal transplant patient 10 years after percutaneous allograft biopsy. Transplant Proc 2008;40:2444-2445

10 Yun GY, Kim SK, Park SK, et al. Asymptomatic renal pseudoaneurysm after percutaneous renal biopsy. Kidney Res Clin Pract 2013; 32(2):87-89

11 Helck A, Hoffmann RT, Sommer WH, et al. Diagnosis, therapy monitoring and follow up of renal artery pseudoaneurysm with contrast-enhanced ultrasound in three cases. Clin Hemorheol Microcirc 2010;46(2-3):127-137

12 Halachmi S, Chait P, Hodapp J, et al. Renal pseudoaneurysm after blunt renal trauma in a pediatric patient: management by angiographic embolization. Urology 2003;61(1):224

13 Inci K, Cil B, Yazici S, et al. Renal artery pseudoaneurysm: complication of minimally invasive kidney surgery. J Endourol 2010; 24(1):149-154

14 Philippou P, Moraitis K, El-Husseiny T, Wazait H, Masood J, Buchholz N. Endovascular covered stenting for the management of post-percutaneous nephrolithotomy renal pseudoaneurysm: a case report. J Med Case Reports 2010;4(1):316

15 Watanabe M, Padua HM, Nguyen HT, Alomari AI. Renal pseudoaneurysm following laser lithotripsy: endovascular treatment of a rare complication. J Pediatr Urol 2010;6(4):420-422
16 Ginat DT, Saad WEA, Turba UC. Transcatheter renal artery embolization: clinical applications and techniques. Tech Vasc Interv Radiol 2009;12(4):224-239

17 Loffroy R, Rao P, Kwak B-K, et al. Transcatheter arterial embolization in patients with kidney diseases: an overview of the technical aspects and clinical indications. Korean J Radiol 2010;11(3):257-268

18 Breyer BN, McAninch JW, Elliott SP, Master VA. Minimally invasive endovascular techniques to treat acute renal hemorrhage. J Urol 2008;179(6):2248-2252, discussion 2253

19 Yang HK, Koh ES, Shin SJ, Chung S. Incidental renal artery pseudoaneurysm after percutaneous native renal biopsy. BMJ Case Rep 2013;2013:bcr2012006537

20 Rivera M, Villacorta J, Jiménez-Alvaro S, Quereda C. Asymptomatic large extracapsular renal pseudoaneurysm following kidney transplant biopsy. Am J Kidney Dis 2011;57(1):175-178

21 Duprey A, Favre J-P, Barral X. Pseudoaneurysms postangioplasty of the renal artery: case reports and review of the literature. Ann Vasc Surg 2009;23(2):258.e1-258.e7

22 Deitch JS, Hansen KJ, Regan JD, Burkhart JM, Ligush J Jr. Infected renal artery pseudoaneurysm and mycotic aortic aneurysm after percutaneous transluminal renal artery angioplasty and stent placement in a patient with a solitary kidney. J Vasc Surg 1998;28(2):340-344

23 Steinway ML, Jankowski JT, Harkey PP, Spirnak JP. Renal artery pseudoaneurysm from blunt abdominal trauma. J Urol 2009; 182(1):314

24 Saad DF, Gow KW, Redd D, Rausbaum G, Wulkan ML. Renal artery pseudoaneurysm secondary to blunt trauma treated with microcoil embolization. J Pediatr Surg 2005;40(11):e65-e67

25 Cohenpour M, Strauss S, Gottlieb P, et al. Pseudoaneurysm of the renal artery following partial nephrectomy: imaging findings and coil embolization. Clin Radiol 2007;62(11):1104-1109

26 Irwine C, Kay D, Kirsch D, Milburn JM. Renal artery embolization for the treatment of renal artery pseudoaneurysm following partial nephrectomy. Ochsner J 2013;13(2):259-263

27 Shapiro EY, Hakimi AA, Hyams ES, Cynamon J, Stifelman M, Ghavamian R. Renal artery pseudoaneurysm following laparoscopic partial nephrectomy. Urology 2009;74(4):819-823

28 Eng M, Vaughan TE, Woo D, Buell JF. Treatment of an extracapsular post-renal biopsy pseudoaneurysm with ultrasound-guided thrombin injection. J Clin Ultrasound 2010;38(4):215-217 